\title{
Materials and Integration Strategies for Modern RF Integrated Circuits
}

\author{
Daniel S. Green ${ }^{1}$, Carl L. Dohrman², Avinash S. Kane ${ }^{2}$, Tsu-Hsi Chang ${ }^{3}$ \\ ${ }^{1}$ U.S. Defense Advanced Research Projects Agency (DARPA), Arlington, VA 22203 \\ ${ }^{2}$ Booz Allen Hamilton, Inc., Arlington, VA 22203 \\ ${ }^{3}$ HetInTec Corp.; Rockville, MD 20850
}

\begin{abstract}
The DARPA Microsystems Technology Office is developing revolutionary materials, devices, and integration techniques for meeting the RF integrated circuit performance requirements for advanced modern RF systems. DARPA is enabling these systems through systematic development of materials and devices, circuits, and integration technologies for compound semiconductors. The DARPA Nitride Electronic NextGeneration Technology (NEXT) program is developing high performance nitride transistors for high-speed RF, analog and mixed signal electronics, thus overcoming the Johnson figure of merit limits to achieving simultaneous high-speed operation and high breakdown voltage. The DARPA Microscale Power Conversion (MPC) program is developing nitride-based technology to enable dynamic envelope-tracking power conversion embedded in RF radiating elements. The DARPA Diverse Accessible Heterogeneous Integration (DAHI) program is developing transistor-scale heterogeneous integration processes to intimately combine advanced compound semiconductor (CS) devices, as well as other emerging materials and devices, with high-density silicon CMOS technology. Taken together, these programs are addressing many of the critical challenges for next-generation RF modules and seek to revolutionize DoD capabilities in this area.
\end{abstract}

Modern RF systems are under consistent pressure to make use of the RF spectrum in increasing sophisticated ways. As the RF spectrum becomes increasingly congested, RF systems must contend with increased interference while achieving frequency agility and spectral efficiency and simultaneously achieving increased data rates. Additionally, as mobile technology becomes more pervasive in both commercial and DoD technology, novel RF systems must work within increasingly limited power budgets. Furthermore, there is a constant push to achieve reduced size and weight for RF systems of all size scales.

Such system requirements create concomitant challenges for RF integrated circuits for which compound semiconductors provide a potential solution. The compound semiconductor (CS) electronics industry has a long history of driving advancements in RF/mixed signal systems. The success of CS materials in these systems is due in large part to the many superior properties of these materials relative to silicon. For example, high electron mobility and peak velocity of InP-based material systems have resulted in transistors with $\mathrm{f}_{\max }$ above $1 \mathrm{THz}[1]$ as well as ultra-high-speed mixed-signal circuits (see, for example, [2]). The wide energy bandgap of $\mathrm{GaN}$ has enabled large voltage swings as well as high breakdown voltage RF power devices [3]. Excellent thermal conductivity of $\mathrm{SiC}$ also makes tens of kilowatt-level power switches possible [4]. Additionally, on-chip high-Q microelectromechanical resonators and switches in various materials, such as AlN, have been demonstrated that potentially can be used for clock references and frequency selective filters [5].

The advantages of CS materials are illustrated in Table 1 below. As indicated by Table 1, compound semiconductors exhibit many superior materials properties relative to silicon technology. However, the modest complexity of CS circuits relative to silicon circuits has limited their deployment in many applications. In particular, $\mathrm{GaN}$ is a material system with an unparalleled combination of high breakdown field, band gap, and thermal conductivity, but with limited integration complexity to date. Overall, it is clear that no materials system excels for all metrics highlighting the benefit to integration.

\begin{tabular}{|c|c|c|c|c|c|c|c|}
\hline Parameter & Why? & Unit & Si & GaAs & ABCS $^{1}$ & $\ln P^{2}$ & $\mathrm{GaN}^{3}$ \\
\hline $\begin{array}{l}\text { Electron } \\
\text { Mobility }\end{array}$ & Carrier velocity & $\begin{array}{c}10^{3} \\
\mathrm{~cm}^{2} / \mathrm{V} \cdot \mathrm{s}\end{array}$ & 1.4 & 8.5 & 40 & 12 & $<1$ \\
\hline$V_{\text {peak }}$ & Transit time & $10^{7} \mathrm{~cm} / \mathrm{s}$ & 1 & 2 & 8 & 2.5 & 2.5 \\
\hline$E_{B K}$ & Voltage swing & $10^{5} \mathrm{~V} / \mathrm{cm}$ & 5.7 & 6.4 & 0.4 & 4 & 40 \\
\hline$E_{g}$ & $\begin{array}{l}\text { Charge } \\
\text { density }\end{array}$ & $\mathrm{eV}$ & 1.12 & 1.42 & 0.35 & 0.74 & 3.4 \\
\hline$\kappa$ & Heat removal & $\mathrm{W} / \mathrm{cm} \cdot \mathrm{K}$ & 1.3 & 0.5 & 0.27 & 0.05 & 2.9 \\
\hline Maturit & $\begin{array}{c}\text { Circuit } \\
\text { Complexity }\end{array}$ & & lent & Good & Limited & Ok & Limited \\
\hline
\end{tabular}

Table 1. Material properties and circuit maturity of various semiconductor technologies. Footnotes: 1 - InAs channel, 2 InGaAs channel, 3 - $\mathrm{SiC}$ substrate.

In order to address the challenges of next-generation RF system requirements, substantial innovation in compound semiconductor materials, devices, circuits, and integration technologies is required. The DARPA Microsystems Technology Office has invested in a number of programs which seek to provide this innovation. The DARPA Nitride Electronic NextGeneration Technology (NEXT) program [6] is 
developing high performance nitride transistors for high-speed RF, analog and mixed signal electronics, thus overcoming the Johnson figure of merit limit to achieving simultaneous high-speed operation and high breakdown voltage. The DARPA Microscale Power Conversion (MPC) program [7] is developing nitridebased technology to enable dynamic envelope-tracking power conversion embedded in RF radiating elements. The DARPA Diverse Accessible Heterogeneous Integration (DAHI) program [8] is developing transistor-scale heterogeneous integration processes to intimately combine advanced compound semiconductor (CS) devices, as well as other emerging materials and devices, with high-density silicon CMOS technology. This paper reviews the latest technical progress of these three DARPA programs.

\section{DARPA NEXT PROGRAM}

The DARPA NEXT program has made significant strides in enhancing the capabilities of $\mathrm{GaN}$ transistor technology to more fully realize GaN's potential for high speed-high breakdown devices, as indicated by its superior Johnson figure of merit [10]. As reported previously [6], NEXT has aggressively pushed the operating speed of GaN HEMTs by simultaneously minimizing carrier transit time, maximizing electron density, reducing access resistances, and optimizing parasitic capacitances with novel device structures.

The DARPA NEXT program goals focus on $\mathrm{GaN}$ enhancement-mode and depletion-mode HEMT performance, yield, uniformity, and reliability. These are shown in Figure 1, with the check marks indicating metrics that have been met by the program. This table indicates the considerable success that the DARPA NEXT performers have achieved in relation to the program goals.

\begin{tabular}{|c|c|c|c|c|c|c|}
\hline & Metric & Unit & Today & Phase I & Phase II & Phase III \\
\hline \multirow{6}{*}{ 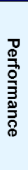 } & D-mode $f_{T}$ & $\mathrm{GHz}$ & 150 & $\sqrt{300}$ & $\sqrt{ } 400$ & 500 \\
\hline & D-mode $f_{\max }$ & $\mathrm{GHz}$ & 200 & $\sqrt{350}$ & $\sqrt{450}$ & 550 \\
\hline & D-mode Johnson FOM ${ }^{(1)}$ & $\mathrm{THz} \cdot \mathrm{V}$ & N/A & $\sqrt{5}$ & $\sqrt{5}$ & $\sqrt{5}$ \\
\hline & E-mode $f_{T}$ & $\mathrm{GHz}$ & 85 & $\sqrt{ } 200$ & $\checkmark 300$ & 400 \\
\hline & E-mode $f_{\max }$ & $\mathrm{GHz}$ & 150 & $\sqrt{ } 250$ & $\sqrt{350}$ & $\sqrt{ } 450$ \\
\hline & E-mode Johnson FOM (1) & $\mathrm{THz} \cdot \mathrm{V}$ & N/A & $\sqrt{5}$ & $\sqrt{5}$ & $\sqrt{5}$ \\
\hline \multirow{2}{*}{$\frac{\widehat{\alpha}}{2}$} & Transistor yield ${ }^{(2)}$ & $\%$ & N/A & $\sqrt{50^{(3)}}$ & $\sqrt{ } 75^{(3)}$ & $95^{(4)}$ \\
\hline & PCM yield (5) & $\%$ & N/A & $\sqrt{ } 30^{(6)}$ & $\sqrt{30}(7)$ & $\sqrt{70^{(8)}}$ \\
\hline \multirow{4}{*}{ 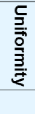 } & $\sigma\left(V_{\text {TH }}\right)^{(9)}$ & $\mathrm{mv}$ & N/A & $\sqrt{50^{(3)}}$ & $\sqrt{40^{(3)}}$ & $\sqrt{30^{(4)}}$ \\
\hline & $\sigma\left(f_{T}\right)^{(9)}$ & $\mathrm{GHz}$ & N/A & $\sqrt{50^{(3)}}$ & $\sqrt{40^{(3)}}$ & $\sqrt{30^{(4)}}$ \\
\hline & $\sigma\left(f_{o s c}\right)^{(9)}$ & $\%$ & N/A & $\sqrt{ } 15^{(6)}$ & $\sqrt{10(7)}$ & $\sqrt{ } 5^{(8)}$ \\
\hline & Degradation Time ${ }^{(10)}$ & Hrs & N/A & $\checkmark>10$ & $\checkmark>100$ & $>1000$ \\
\hline
\end{tabular}

Figure 1. DARPA NEXT program goals. Check marks indicate goals which have been met.

The current status of HEMT operating speed in the DARPA NEXT program is shown in Figure 2. Aggressive device scaling has resulted in enhancementand depletion-mode GaN HEMTs with dramatically improved unity-gain cutoff frequency $\left(\mathrm{f}_{\mathrm{T}}\right)$ and maximum oscillation frequency $\left(f_{\max }\right)$ as compared to the state-of-the-art prior to the program. Additionally,
NEXT has aggressively improved device yield and circuit complexity, achieving a 501-stage ring oscillator using both enhancement-mode and depletion-mode HEMTs on a single substrate.

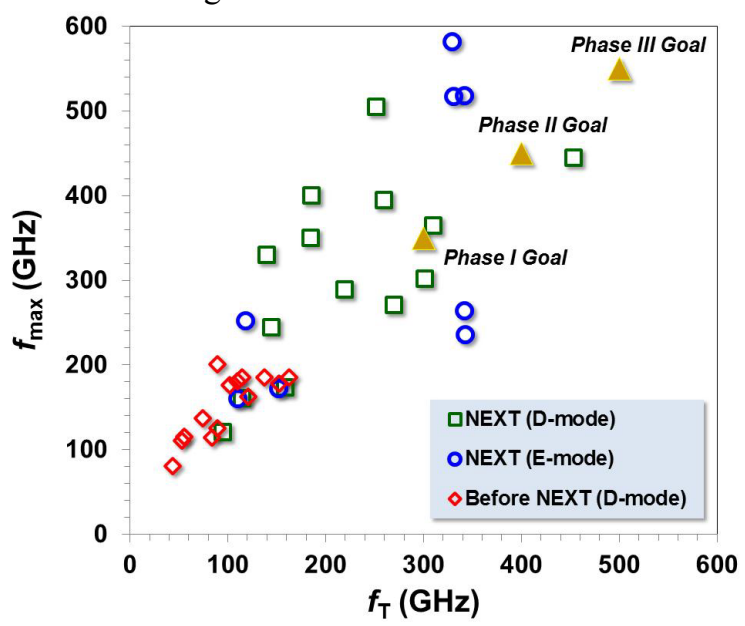

Figure 2. Plot of $f_{\max }$ versus $f_{T}$ for GaN HEMTs developed in the DARPA NEXT program. State-of-theart GaN HEMTs developed before NEXT shown for comparison.

\section{DARPA MPC PROGRAM}

The DARPA MPC program has focused on leveraging recent progress in $\mathrm{GaN}$ transistor technology to develop GaN MMIC-based RF power amplifiers which utilize envelope-tracking to achieve very high efficiency at X-band frequencies. As reported previously [7], the program envisions co-designing a MMIC power amplifier with a dynamic voltage power supply modulator consisting of a very fast GaN power switch.

MPC program participants have made substantial progress in advancing the state-of-the-art in envelopetracking power supply technology. Figure 3 plots power-added efficiency versus operating frequency for state-of-the-art power amplifiers. Results from the MPC program show the efficiency increase from $20 \%$ to over $50 \%$ at $10 \mathrm{GHz}$. Similarly, Figure 4, which plots efficiency versus envelope bandwidth, shows that the MPC program achieved a $500 \mathrm{MHz}$ RF envelope bandwidth with an efficiency of $40 \%$ or greater, which is more than an order of magnitude increase over the state of the art $(20 \mathrm{MHz})$. 


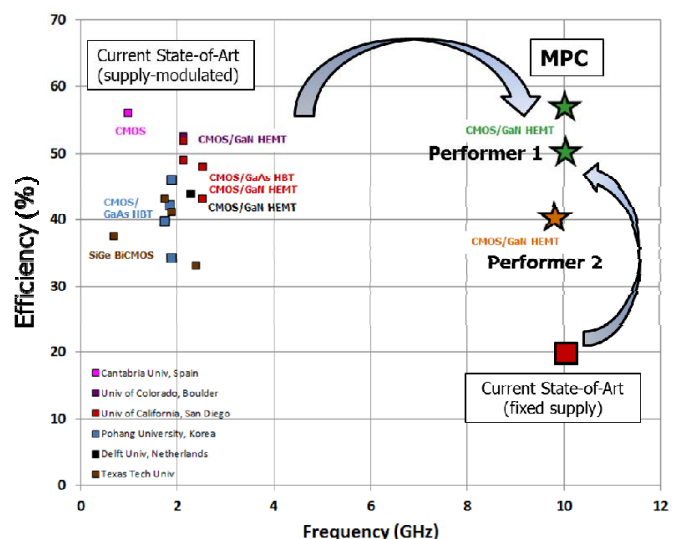

Figure 3. Plot of power-added efficiency versus output frequency for state-of-the-art power amplifiers.

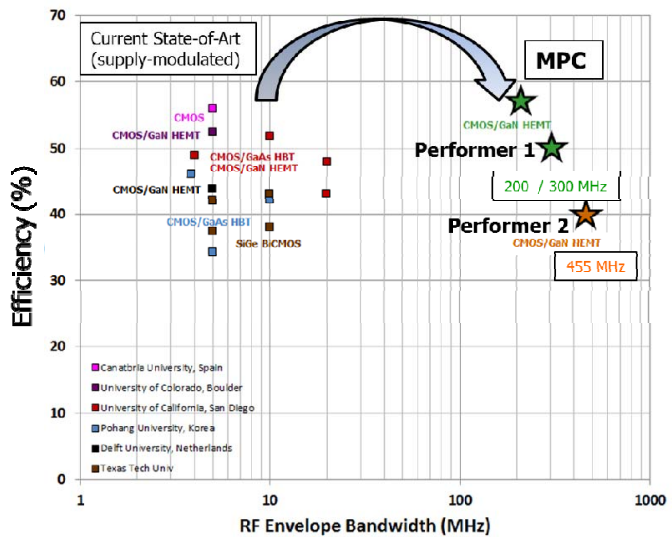

Figure 4. Plot of power-added efficiency versus RF envelope bandwidth for state-of-the-art envelopetracking power amplifiers.

\section{DARPA DAHI PROGRAM}

The preceding sections have highlighted many of the advantages of CS materials for RF modules. However, despite the advantages of CS materials, Si CMOS-based technologies have increasingly been employed in highperformance $\mathrm{RF} /$ mixed signal systems. These technologies have leveraged the enormous investments in digital CMOS device scaling and process development to achieve tremendous levels of complexity and integration, while also demonstrating far higher levels of yield and manufacturability than any CS technology. The integration density of Si-based technologies has enabled novel on-chip digital correction and linearization techniques (for example, [9]), producing excellent RF and mixed-signal circuit performance despite the limitations of silicon's material properties.

Such correction techniques have the potential to produce dramatic RF and mixed-signal performance improvements in CS electronics as well; however, CS technologies lack the integration density and yield to implement these circuit concepts. Given these trends, it is our view that the future of high-performance RF and mixed-signal electronics lies in the integration of CS materials with silicon technology in a way that will allow the advantages of the two technology types to be optimally combined.

Figure 5 illustrates the potential impact of heterogeneous integration in RF/mixed signal systems, using a representative transceiver as an example. Essentially all major components in a typical transceiver can potentially benefit from the reduced parasitics of heterogeneous integration, utilizing the benefits of highperformance CS materials with the control and calibration capabilities of Si CMOS.

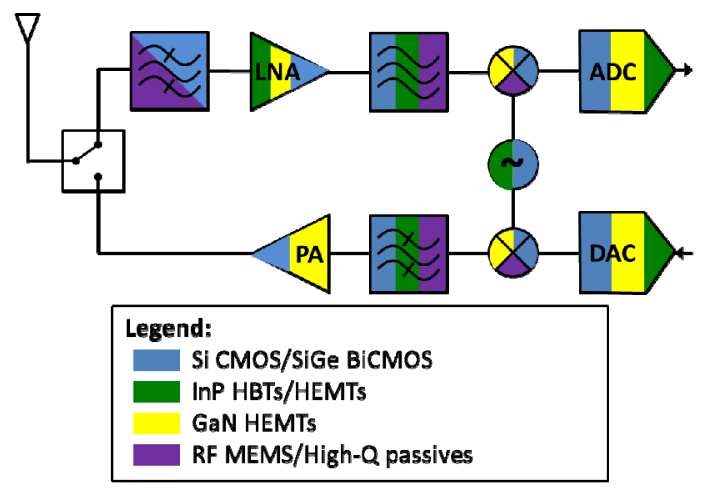

Figure 5. Diagram of a representative transceiver illustrating the preferred set of device technologies for optimal performance of each transceiver component.

To that end, the DARPA DAHI program is developing transistor-scale heterogeneous integration processes to intimately combine advanced compound semiconductor (CS) devices, as well as other emerging materials and devices, with high-density silicon CMOS technology. The ultimate goal of DAHI is to establish a manufacturable, accessible foundry technology for the monolithic heterogeneous co-integration of diverse (e.g., electronic, photonic, MEMS) devices, and complex silicon-enabled architectures, on a common substrate platform for defense and commercial users. This capability will not only have significant impacts on the performance of both military and commercial microsystems, but it also represents a new paradigm for the CS electronics community.

One element of DAHI, the Compound Semiconductor Materials on Silicon (COSMOS) thrust, has demonstrated three different approaches (shown in Figure 3) to achieving InP BiCMOS integrated circuit technology featuring InP HBTs and deep submicron Si CMOS [12][13][14] for RF and mixed signal circuits.

DAHI/COSMOS performers have demonstrated complex heterogeneously integrated mixed-signal circuit designs, including digital-to-analog converters (DACs) with unprecedented SFDR performance in the $\mathrm{GHz}$ output frequency regime [15] as well as revolutionary ultra-wide band ADCs. The ADC design employs a time-interleaved architecture utilizing InP 
HBTs for track-and-hold circuitry and 130nm Si CMOS to provide the sub-ADCs and the circuitry required for complex time-interleaving. This ADC represents the most complex heterogeneously integrated circuit to date, with $\sim 1000 \mathrm{InP}$ HBTs, $\sim 16,000 \mathrm{Si}$ HBTs, and $2500 \mathrm{Si}$ MOSFETs, with more than 1800 heterogeneous interconnects between the InP HBT chiplet and the Si base chip. This approach has resulted in an ultra-wide band $\mathrm{ADC}$ with state-of-the-art signal-to-noise-anddistortion ratio (SINAD) of over $30 \mathrm{~dB}$ across frequency ranges of $2.75-8.75 \mathrm{GHz}$ and $14.25-20.25 \mathrm{GHz}$. This performance level is on par with the most advanced ADCs currently demonstrated [16], which required the use of $32 \mathrm{~nm}$ CMOS technology (as compared to the $130 \mathrm{~nm}$ CMOS used by the DAHI/COSMOS ADC). It is expected that the combination of InP HBTs heterogeneously integrated with an advanced CMOS node would enable dramatically improved SINAD at higher frequencies, far in excess of the current state-ofthe-art. DAHI performers have also demonstrated the world's first $\mathrm{GaN}+\mathrm{CMOS} \mathrm{RF}$ amplifier using monolithic heterogeneous integration of GaN HEMTs with Si pMOS gate bias control [17].

Recently, a new DAHI Foundry Technology thrust was initiated [18] to advance the diversity of heterogeneous device and materials available in a silicon-based platform. This foundry will seek to include a wider array of materials and devices (including, GaN and MEMS technologies) with complex silicon-enabled (e.g. CMOS) architectures and thermal management structures on a common silicon substrate platform. The goal of the DAHI Foundry Technology thrust is to develop a mature, reliable heterogeneous integration technology in a cost-effective foundry.

\section{ACKNOWLEDGEMENT}

The authors would like to thank the program participants in the DARPA NEXT, MPC, and DAHI program. The authors also thank the NEXT, MPC, and DAHI government team members for their support. Finally, the authors thank the original DARPA program managers: John Albrecht (NEXT and MPC), Sanjay Raman (DAHI), and Mark Rosker (COSMOS).

The views, opinions, and/or finding contained in this article/presentation are those of the author(s)/ presenter(s) and should not be interpreted as representing the official views or policies, either expressed or implied, of the Defense Advanced Research Project Agency or the Department of Defense.

\section{REFERENCES}

[1] R. Lai, et al, "Fabrication of InP HEMT Devices with Extremely High Fmax", 2008 International Conference on InP and Related Materials, Versailles, France.

[2] S.E. Turner, D.E. Kotecki, "Direct Digital Synthesizer With Sine-Weighted DAC at 32-GHz Clock Frequency in InP DHBT Technology," IEEE J. Solid-State Circuits, Vol. 41, No. 10, 2006, pp. 2284-90.

[3] Y.F. Wu; M. Moore, A. Saxler, T. Wisler, P. Parikh, "40W/mm Double Field Plated GaN HEMTs," 64th Device Research Conference, 2006, pp. 151-152.

[4] Cree's SiC Schottky Diode Chip CPW2-1200-S050B.

[5] Crespin, E. R., et al., "Fully integrated switchable filter banks," 2012 IEEE MTT-S, pp.1-3, 17-22 June 2012.

[6] Albrecht, J.D.; Tsu-Hsi Chang; Kane, AS.; Rosker, M.J., "DARPA's Nitride Electronic NeXt Generation Technology Program," 2010 IEEE C.S. Integrated Circuit Sym. (CSICS), pp.1-4, 3-6 Oct. 2010.

[7] Albrecht, J.D.; Kane, A; Tsu-Hsi Chang, "DARPA's Microscale Power Conversion Program," 2012 IEEE Compound Semiconductor Integrated Circuit Symposium (CSICS), pp.1-4, 14-17 Oct. 2012.

[8] Green, D. S., Dohrman, C. L., \& Chang, T. H., "The DARPA Diverse Accessible Heterogeneous Integration (DAHI) Program: Status and Future Directions." 2014 Compound Semiconductor Manufacturing Technology conference (CS MANTECH), 2014.

[9] Adrian Tang, et al. , "A Low Overhead Self-Healing Embedded System for Ensuring High Performance Yield and Long- Term Sustainability of a $60 \mathrm{GHz} 4 \mathrm{Gbps}$ Radioon-a-Chip", 2012 ISSCC Digest, 2012.

[10] E.O. Johnson, "Physical limitations on frequency and power parameters of transistors", RCA Review, vol. 26, pp. 163-177, 1965.

[11] Fang, S. F.; Adomi, K.; Iyer, S.; Morkoç, H.; Zabel, H.; Choi, C.; Otsuka, N., "Gallium arsenide and other compound semiconductors on silicon" J. Appl. Phys., 68, R31-R58 (1990).

[12] A. Gutierrez-Aitken, et al., "Advanced heterogeneous integration of InP HBT and CMOS Si technologies for high performance mixed signal applications," IEEE Microwave Symposium Tech. Dig., pp. 1109-1112, 2009.

[13] T.E. Kazior, et al., "A high performance differential amplifier through the direct monolithic integration of $\mathrm{InP}$ HBTs and $\mathrm{Si}$ CMOS on silicon substrates," IEEE Microwave Symposium Tech. Dig., pp. 1113-1116, 2009.

[14] J.C. Li, et al., "Heterogeneous wafer-scale integration of 250nm, 300GHz InP DHBTs with a 130nm RF-CMOS technology," IEDM Tech. Dig., pp. 944-946, 2008.

[15] Oyama, B., et al. "InP HBT/Si CMOS-Based 13-Bit $1.33 \mathrm{Gsps}$ Digital-to-Analog Converter with $>70 \mathrm{~dB}$ SFDR," 2012 CSICS Tech. Dig., pp.1-4, 2012.

[16] Kull, L., et al., "A 90GS/s 8b 667mW 64× interleaved SAR ADC in 32nm digital SOI CMOS," 2014 ISSCC Proc., pp.378-9, 2014.

[17] W. E. Hoke, et al. "Monolithic integration of silicon CMOS and GaN transistors in a current mirror circuit", $J$. Vac. Sci. Tech. B 30(2), pp. 02B101-1-6, 2012.

[18] http://www.darpa.mil/Our_Work/MTO/Programs/DAHI/ DAHI_Foundry_Technology.asp. 